\title{
Evaluation Method of Enhanced Fluid Effect on offshore Oilfield
}

\author{
Zeng yuan-fang, Li hao, Song zhi-cong, Li ming-jun \\ CNOOC -Ltd_Zhanjiang. Zhanjiang, 524057, China
}

\author{
*Corresponding Author: Zeng yuan-fang, CNOOC -Ltd_Zhanjiang. Zhanjiang, 524057, China
}

\begin{abstract}
The relative permeability curve of oil and water has a very important role in the development of oil and gas fields. It can be used to analyze the law of oil production, calculate the oil production and flow ratio, and determine the saturation distribution of oil and water in reservoir and the location of oil and water contact surface. Based on the samples of 19-1 and 8-3 blocks in Wenchang Oilfield, the seepage curves of oil - water two - phase were calculated and the characteristics of the seepage curves under different flow rates were obtained.
\end{abstract}

Keywords: relative permeability, Wenchang Oilfield, velocity

\section{INTRODUCTION}

Relative permeability curve data is an important part of oilfield basic research, especially the reservoir engineering (calculation and development indicators, dynamic analysis, etc.) and reservoir numerical simulation engineering calculation work is very important parameters. However, when the oil field to extract measures, the flow rate increases, the water content also increases, this situation and the actual seepage does not meet the law. In order to explain the actual situation of this field, the relationship between velocity and infiltration is studied. Leverett first proposed that during the laboratory experiment, the measured seepage curve is related to the velocity [1]. In this paper, by analyzing the seepage and flooding efficiency under different flow rates, the experimental data are combined with the actual situation, and the seepage characteristics under different flow rates are obtained, which is of great significance to the reservoir engineering follow-up work.

\section{TeSt The Experimental DeVice ANd Principle}

It is of great significance to study the oil infiltration under different flow conditions. In this paper, the study of the reservoirs in the 19-1 and 8-3 blocks of Wenchang Oilfield is studied. The experimental study is carried out to study the dynamic relative permeability experiment. Figure 2 shows the internal design of the cabinet.

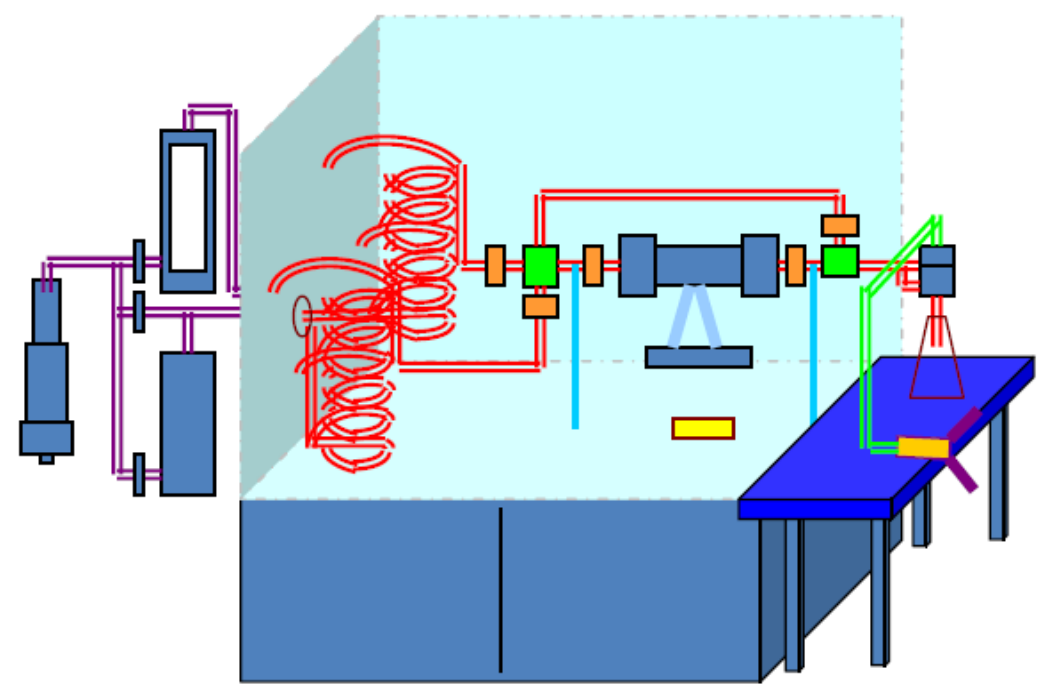

Figure1. Relative permeability test box internal design diagram 
The experimental scheme for determining the relative permeability was established. The experimental procedure was as follows:

(1) The model vacuum, into the saturated formation of water;

(2) The model into the incubator, emptying the valve and pipeline, and connected to the process, in 20 ${ }^{\circ} \mathrm{C}$ constant temperature conditions, for 10 hours;

(3) Into the saturated crude oil, record the output side of the water production;

(4) After aging for 24 hours, the container filled with experimental water was evacuated for 15 minutes;

(5) The formation of water into the model, the use of unsteady constant pressure method for the experiment;

(6) record the output side of the oil production and water production data, the experiment to the export side of the water content of more than $99.95 \%$ or cumulative water injection when the end of 500PV;

(7) To draw the relative permeability curve;

(8) Change the displacement speed, repeat the above process.

The experiment uses three core samples of Wenchang 19-1N-1, and two samples of Wenchang 8-3-2 wells. The experimental fluid was $19-1 \mathrm{~N}-1$ well, oil viscosity: $1.41 \mathrm{mPa} \cdot \mathrm{s}, 19-1 \mathrm{~N}-1$ well, water viscosity: $1.01 \mathrm{mPa} \cdot \mathrm{s}, 8-3-2$ well, oil viscosity: $2.2 \mathrm{mPa} \cdot \mathrm{s}, 8-3-2$ well, water viscosity: $0.69 \mathrm{mPa} \cdot \mathrm{s}$.

\section{TeSt Results}

According to similar criteria, as follows:

$$
\begin{aligned}
& \frac{P^{\prime} t^{\prime}}{\left(L^{\prime}\right)^{2} \mu^{\prime}}=\frac{P^{\prime \prime} t^{\prime \prime}}{\left(L^{\prime \prime}\right)^{2} \mu^{\prime \prime}} \\
& \frac{P^{\prime}}{L^{\prime}}=\frac{P^{\prime \prime}}{L^{\prime \prime}} \\
& \frac{\rho^{\prime} g^{\prime} L^{\prime}}{\rho^{\prime}}=\frac{\rho^{\prime \prime} g " L^{\prime \prime}}{\rho^{\prime \prime}} \\
& \frac{P^{\prime}}{\sigma^{\prime}(\cos \theta)^{\prime}}=\frac{P^{\prime \prime}}{\sigma^{\prime \prime}(\cos \theta)^{\prime \prime}}
\end{aligned}
$$

The scale ratio: 2000; velocity scale: 4000000 , get the actual production data and indoor experiment conversion, as shown in Table 1, the actual production data and indoor experiment, as shown in Table 1 , The actual flow rate and model flow rate conversion, as shown in Table 2.

Table1. Conversion between indoor and actual production

\begin{tabular}{|c|c|c|}
\hline Production targets & Reservoir value & Physical simulation values \\
\hline Length & $300 \mathrm{~m}$ & $15 \mathrm{~cm}$ \\
\hline Crude oil viscosity & $0.914 \mathrm{mPa} \cdot \mathrm{s}$ & $0.914 \mathrm{mPa} \cdot \mathrm{s}$ \\
\hline Water viscosity & $0.5 \mathrm{mPa} \cdot \mathrm{s}$ & $0.5 \mathrm{mPa} \cdot \mathrm{s}$ \\
\hline Oil density & $0.7158 \mathrm{~g} / \mathrm{cm}^{3}$ & $1 \mathrm{~g} / \mathrm{cm}^{3}$ \\
\hline Water density & $1 \mathrm{~g} / \mathrm{cm}^{3}$ & $21.1 \%$ \\
\hline Porosity & $21.1 \%$ & $865.5 \mathrm{mD}$ \\
\hline Permeability & $865.5 \mathrm{mD}^{3}$ & $0.017 \mathrm{ml} / \mathrm{min}-0.52 \mathrm{ml} / \mathrm{min}$ \\
\hline Water injection strength & $100-3000 \mathrm{~m}^{3} / \mathrm{d}$ & $\mathrm{m}$ \\
\hline
\end{tabular}

8-3A oil field A1H well in March 1, 2013 to extract the liquid, before the maximum flow rate of about $0.6 \mathrm{~m} /$ day (converted to the core experiment flow rate of $0.2 \mathrm{ml} / \mathrm{min}$ ), the maximum flow rate after extraction is about $2.5 \mathrm{~m} /$ day $(0.85 \mathrm{ml} / \mathrm{min})$.

19-1B oil field B1 wells in October 9, 2009 to extract measures, the maximum flow rate before extraction of about $1.8 \mathrm{~m} /$ Day (converted to the core experiment flow rate of $0.85 \mathrm{ml} / \mathrm{min}$ ), the 
maximum flow rate after extraction of about $5.7 \mathrm{~m} /$ day $(1.93 \mathrm{ml} / \mathrm{min})$.

The maximum flow rate of the $\mathrm{B} 4 \mathrm{H}$ wells in the $8-3 \mathrm{~B}$ field was about $1.8 \mathrm{~m} /$ day (the flow rate was $0.61 \mathrm{ml} / \mathrm{min}) .4 .2 \mathrm{~m} /$ day $(1.43 \mathrm{ml} / \mathrm{min})$ as shown in Figure 2,3.

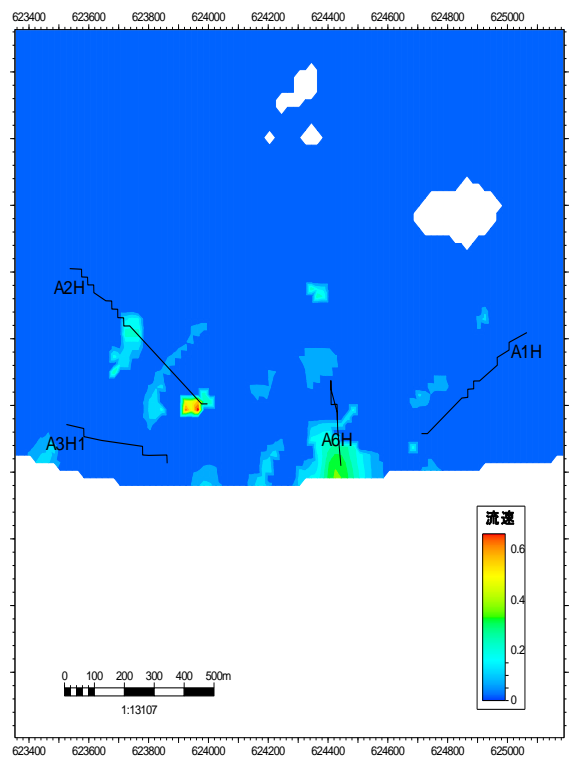

Figure2. 8-3A oil field AlH wells before the flow chart

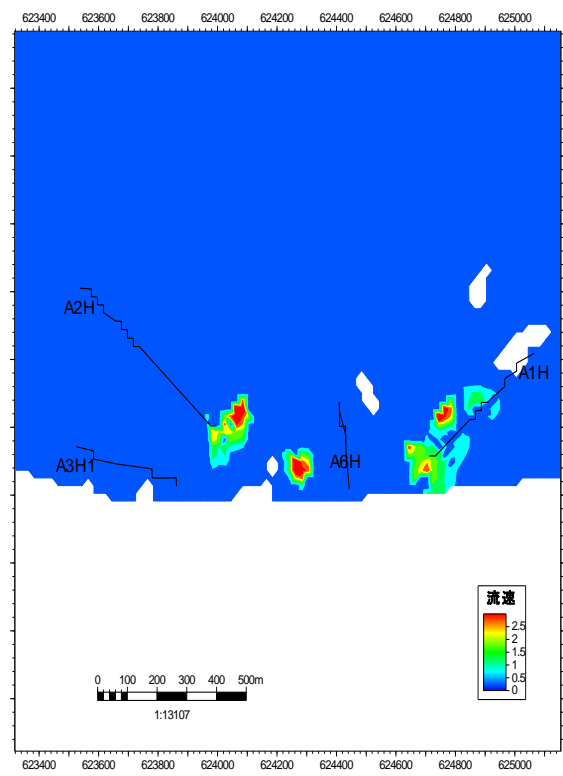

Figure3. 8-3A oil field AlH wells after the flow chart

19-1A oil field May 1, 2015 to extract the measures before the maximum flow rate of about $4.6 \mathrm{~m} /$ day (converted to the core experiment flow rate of $1.56 \mathrm{ml} / \mathrm{min}$ ), the maximum flow rate of about 8.8 $\mathrm{m} /$ Day $(2.98 \mathrm{ml} / \mathrm{min})$.

Table2. Actual flow rate and model flow rate conversion

\begin{tabular}{|c|c|c|}
\hline Test speed & Actual flow rate & Model flow rate \\
\hline 1 & $800 \mathrm{~m}^{3} / \mathrm{d}$ & $0.14 \mathrm{ml} / \mathrm{min}$ \\
\hline 2 & $1200 \mathrm{~m}^{3} / \mathrm{d}$ & $0.21 \mathrm{ml} / \mathrm{min}$ \\
\hline 3 & $1600 \mathrm{~m}^{3} / \mathrm{d}$ & $0.28 \mathrm{ml} / \mathrm{min}$ \\
\hline 4 & $2000 \mathrm{~m}^{3} / \mathrm{d}$ & $0.35 \mathrm{ml} / \mathrm{min}$ \\
\hline 5 & $3000 \mathrm{~m}^{3} / \mathrm{d}$ & $0.52 \mathrm{ml} / \mathrm{min}$ \\
\hline
\end{tabular}

Considering the similarity criterion and numerical simulation method, considering the operability of the experiment, five flow rates $(\mathrm{ml} / \mathrm{min})$ were determined: $0.125,0.25,0.5,1,2$. The relative permeability curves of five flow rates under different flow rates were measured by experiments. 


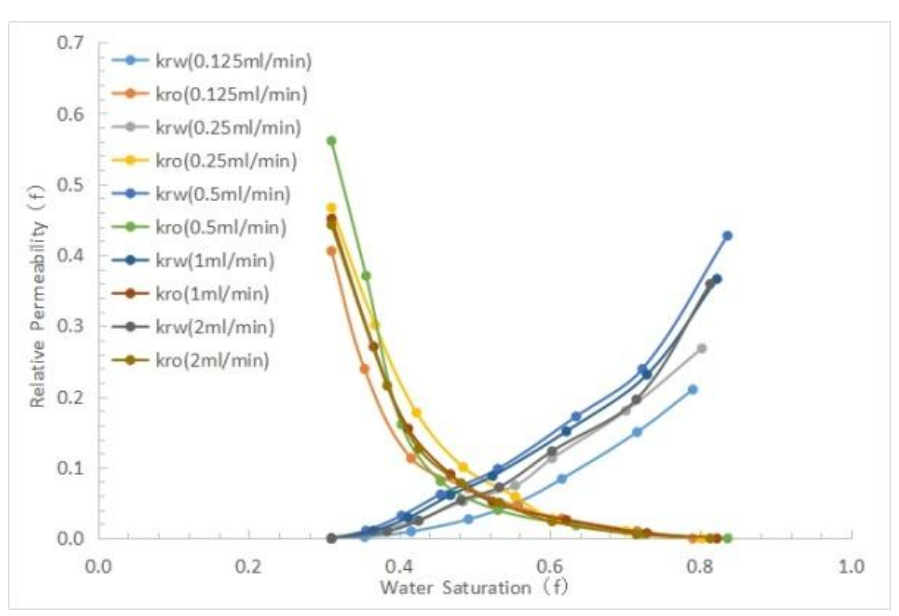

Figure4. 19-1N-1A-1-7 core percolation curve (permeability: $485 \mathrm{md}$ )

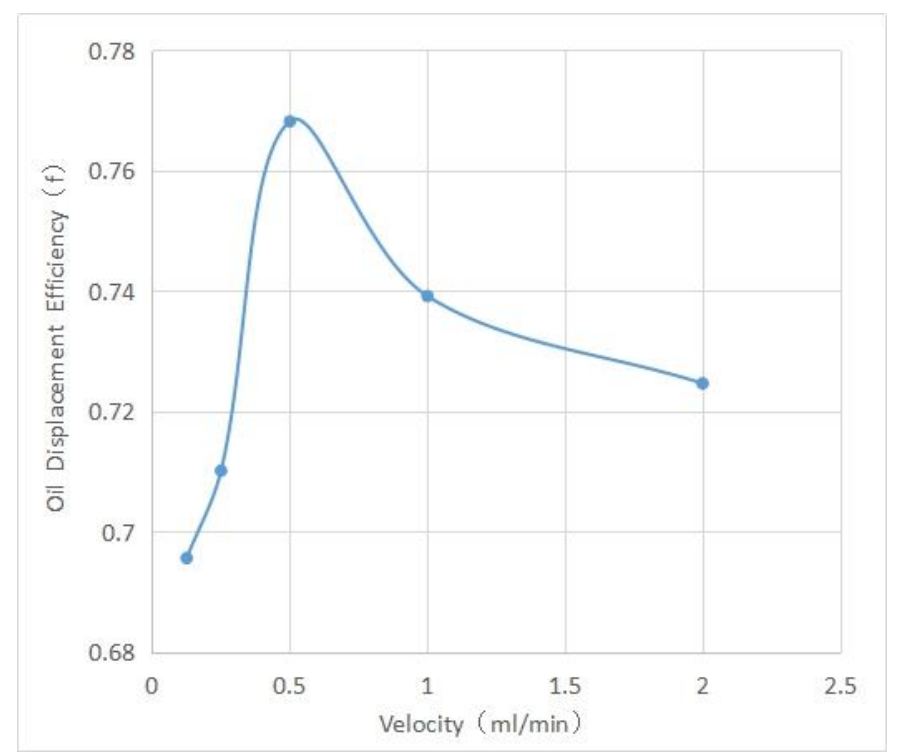

Figure5. Relationship between core flow rate and oil displacement efficiency of 19-1N-1A-1-7 (permeability: $485 \mathrm{md})$

It can be seen from Figure 4 that the oil-water relative permeability curves of the core of 19-1N-1A-17 show the following characteristics: the range of the two-phase permeable area is wide; the saturated water saturation is low, the original oil saturation is low; The relative decrease rate of the relative permeability decreases slowly and the relative permeability of the water phase is lower, showing a concave type. At the flow rate of $0.5 \mathrm{ml} / \mathrm{min}$, the maximum oil displacement efficiency.

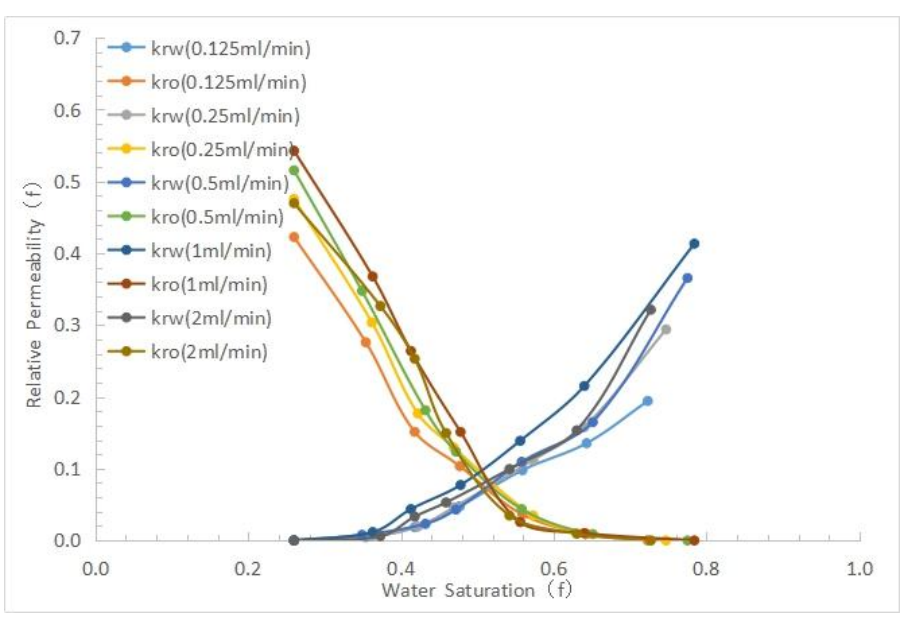

Figure6. 19-1N-1A-1-8 core percolation curve (permeability: 1255md) 


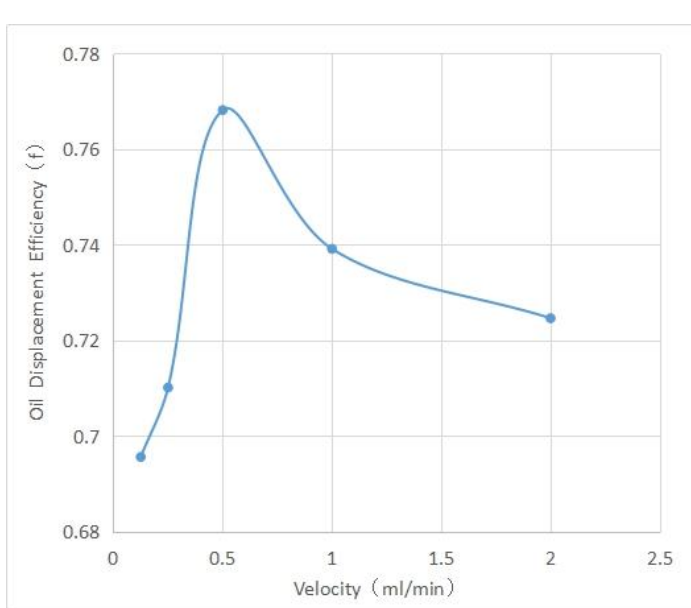

Figure7. Relationship between core flow rate and displacement efficiency of 19-1N-1A-1-8 (permeability: $1255 m d)$

It can be seen from Figure 6, 19-1N-1A-1-8 core oil and water relative permeability curve shows the following characteristics: two-phase permeable area is wide; irreducible water saturation, the original oil saturation is low; The relative permeability of the relative decline rate; water phase relative permeability of the final value is low, showing a concave type. Similarly, at a flow rate of $0.5 \mathrm{ml} /$ min, the oil displacement efficiency is greatest.

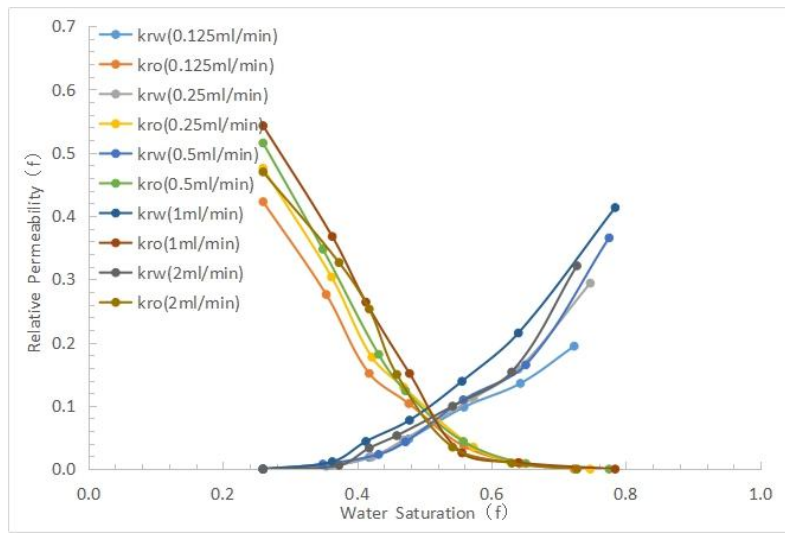

Figure8. 19-1N-1A-1-9 core percolation curve (permeability: 2681md)

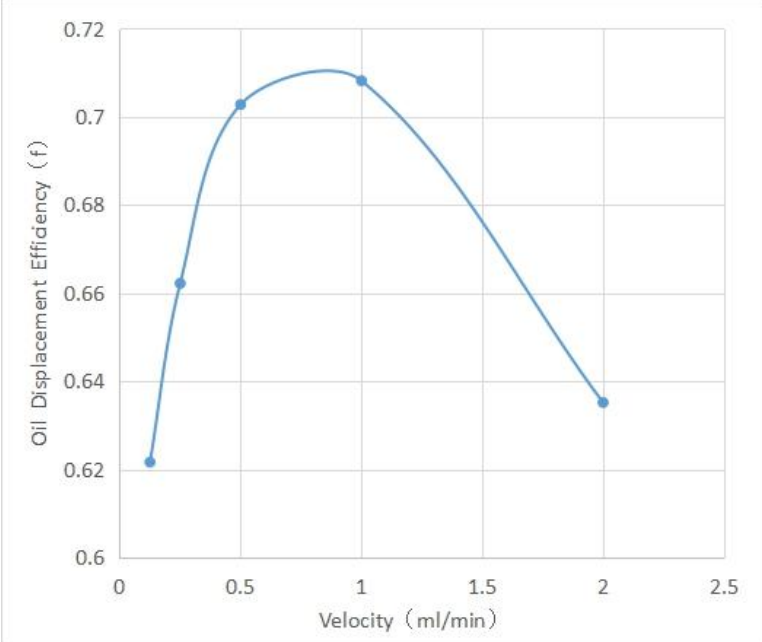

Figure9. Relationship between core flow rate and displacement efficiency of 19-1N-1A-1-9 (Permeability: 2681md)

It can be seen from Figure 8, 19-1N-1A-1-9 core oil and water relative permeability curve shows the following characteristics: two-phase permeable area is wide; irreducible water saturation, the original oil saturation is low; Phase relative permeability decreased slightly faster; the relative permeability of 
water phase is lower, showing a concave type. At the flow rate of about $0.7 \mathrm{ml} / \mathrm{min}$, the oil displacement efficiency is greatest.

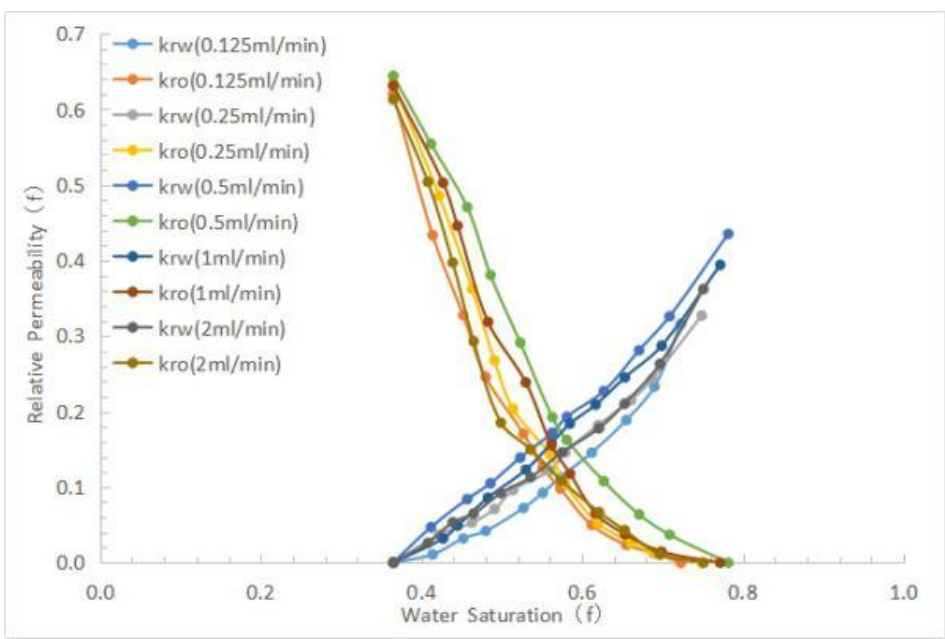

Figure10. 8-3-2-1 core percolation curve (permeability: 962md)

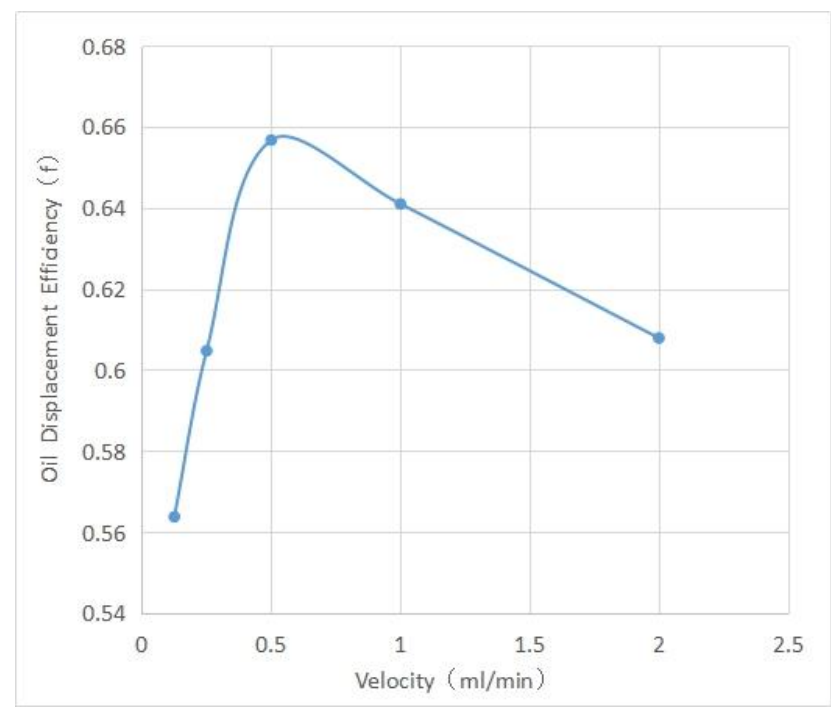

Figure11. 8-3-2-1 core flow rate and displacement efficiency (permeability 962md)

It can be seen from Figure 10, 8-3-2-1 core oil and water relative permeability curve shows the following characteristics: two-phase permeable area narrow; irreducible water saturation, the original oil saturation is low; The rate of decline in permeability is faster; the relative permeability of water phase is lower, showing a concave type. At a flow rate of about $0.5 \mathrm{ml} / \mathrm{min}$, the oil displacement efficiency is greatest.

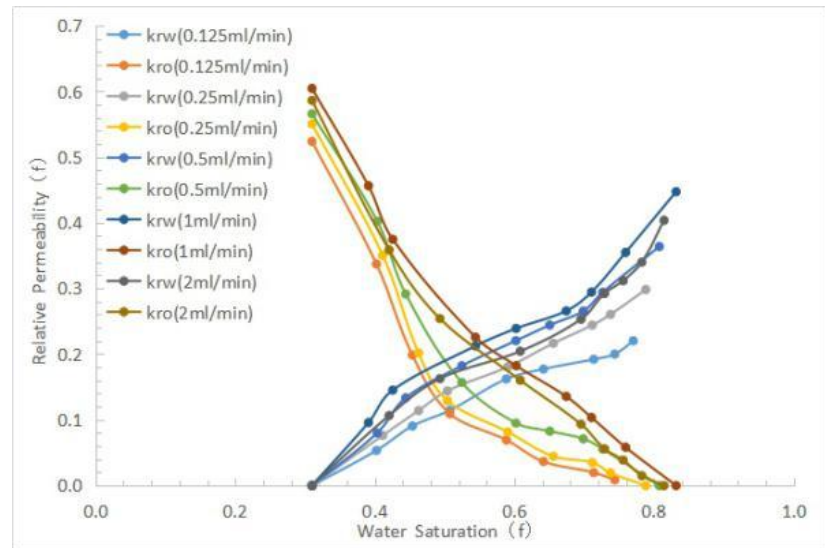

Figure12. 8-3-2-2 core percolation curve (permeability 1566md) 


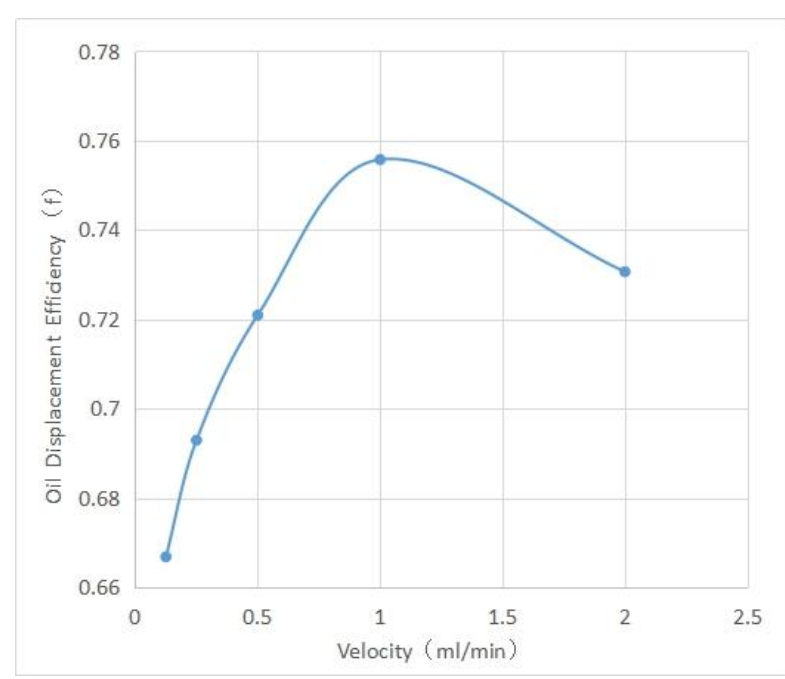

Figure13. 8-3-2-2 core flow rate and displacement efficiency (permeability 1566md)

It can be seen from Fig. 12 that the relative permeability curves of oil and water in the core of 8-3-2-2 show the following characteristics: the range of the two-phase permeation zone is narrow; the irreducible water saturation is high, the original oil saturation is low; The rate of decline of permeability is faster; the relative value of relative permeability of water phase is lower, the part of which is concave and partly convex. At a flow rate of about $1 \mathrm{ml} / \mathrm{min}$, the oil displacement efficiency is greatest.

For the development of oilfield, the relationship between water content and recovery degree is an important means to evaluate the development effect and predict the recovery rate. The relative permeability curve is based on the important seepage foundation of its theoretical relationship [2].

According to the Bernoulli sub-flow equation [3], crude oil viscosity $0.914 \mathrm{mPa} \cdot \mathrm{s}$, water viscosity $0.5 \mathrm{mPa} \cdot \mathrm{s}$, ignoring the capillary pressure and neutral influence, according to the diversion equation can be obtained from the water content and the degree of recovery curve.

When the extraction measures, the flow rate increases, moisture content also changes, then need to consider the relative permeability changes. Taking the $\mathrm{A} 1 \mathrm{H}$ well in the 8-3A well as an example, the relationship between the actual water content and the degree of recovery is shown in Fig12.The results of the seepage test are basically consistent with the actual oil field.

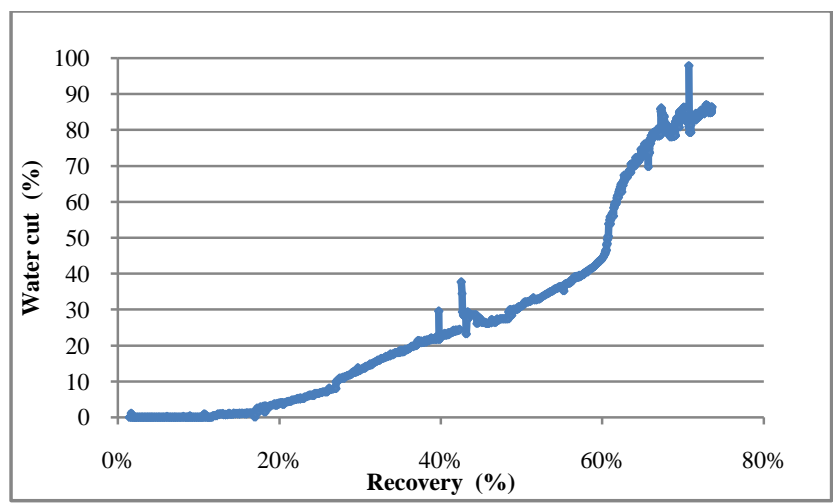

Figure12. 8-3A-A1H water content and the degree of recovery curve

\section{RESULTS ANALYSIS}

According to the experimental data can be obtained under different flow conditions, the permeability curve has the following characteristics:

(1) When the permeability is constant, the higher the flow rate, the higher the relative permeability of the water phase, the lower the relative permeability of the oil phase. However, after a certain limit, the relative permeability of the water phase decreases and the relative permeability of the oil phase decreases. 
(2) when the permeability is constant, the greater the flow rate, the higher the efficiency of oil displacement, but more than a certain limit, the oil discharge efficiency. When the flow rate is constant, the peak of the corresponding displacement efficiency is basically the same.

(3) According to the characteristics of different flow rate seepage curves, the reservoir seepage curve of Wenchang Oilfield is affected by the influence of capillary force and the influence of viscosity. In the flow rate is less than $1 \mathrm{ml} / \mathrm{min}$ (the actual situation: $3 \mathrm{~m} /$ day) range, the infiltration curve is mainly affected by the capillary force; in the flow rate is greater than $1 \mathrm{ml} / \mathrm{min}$, the infiltration curve is mainly affected by the viscosity of the impact.

(4) In the later stage of high water content, the relative permeability of water phase increases rapidly with the increase of water saturation, and the relative permeability of oil phase decreases rapidly.

\section{REFERENCES}

[1] Xiong Jun, Liu Jian, Liu Jianjun et al. Theory of water vapor two-phase percolation based on BuckleyLeverett equation [J]. Liaoning Engineering University Science Edition), 2007, 26 (2): 213-215.

[2] Wang Xiaolin, Yu Lijun, Tang Wei, et al. Relationship between Moisture Content and Recovery Degree in Extraordinary Water Cut Stage [J]. Special Oil and Gas Reservoirs, 2015, 22 (5): 104- 06.

[3] Zhao Changyou. Bernoulli equation and its application [J]. Journal of Chizhou University, 2014 (6): 48-49.

Citation: Zeng yuan-fang et.al.,(2018). Evaluation Method of Enhanced Fluid Effect on offshore Oilfield, International Journal of Petroleum and Petrochemical Engineering (IJPPE), 4(1), pp.87-94, DOI: http://dx.doi.org/10.20431/2454-7980.0401010

Copyright: () 2018 Zeng yuan-fang, (2018). This is an open-access article distributed under the terms of the Creative Commons Attribution License, which permits unrestricted use, distribution, and reproduction in any medium, provided the original author and source are credited 